\title{
The evaluation of chatbot as a tool for health literacy education among undergraduate students
}

\author{
Nur Azlina Mohamed Mokmin ${ }^{1}$ (D) - Nurul Anwar Ibrahim ${ }^{1}$
}

Received: 10 January 2021 / Accepted: 6 April 2021 / Published online: 25 May 2021

(c) The Author(s), under exclusive licence to Springer Science+Business Media, LLC, part of Springer Nature 2021

\begin{abstract}
This study discussed and evaluated the usefulness, performance, and technology acceptance of a chatbot developed to educate users and provide health literacy. A semi-structured interview and analytic sessions were provided on Google Analytics dashboard, and the users' acceptance toward the technology was measured using the Unified Theory of Acceptance and Use of Technology 2 (UTAUT2). A total of 75 undergraduate students were involved over a total period of two months. Each respondent explored the health chatbot actively to get advice from it with a phrase that matched the chatbot's intents via mobile devices. The evaluation results showed that $73.3 \%$ of the respondents found that the chatbot can help understand several health issues and provide a good conversation. The performance evaluation also showed that the chatbot contributed a low percentage of exit, where less than $37 \%$ of users exited the application. The overall assessment showed that the developed chatbot has a significant potential to be used as a conversational agent to increase health literacy, especially among students and young adults. However, more research should be done before the technology can replace humans in a real setting.
\end{abstract}

Keywords Chatbot · Health literacy · Education · Analytics · UTAUT2 · Undergraduate $\cdot$ Analytics

Nur Azlina Mohamed Mokmin nurazlina@usm.my

Nurul Anwar Ibrahim nurulanwar@usm.my

1 Centre of Instructional Technology and Multimedia, Universiti Sains Malaysia, Penang, Malaysia 


\section{Introduction}

Health literacy refers to the degree of a person obtaining and understanding health conditions and information related to the term before making certain judgments or decisions (Selden et al., 2000). Failures in providing health education can lead to low health literacy. The increase in health literacy level leads to better healthrelated knowledge, improved adherence to treatment, smoother patient and health provider communication, improved mental health, and increased patient satisfaction. According to Kickbusch et al. (2013), health literacy is measured by health care, disease prevention, and health promotion. Neter and Brainin (2019), in their study, stated that low health literacy could be associated with several problems related to health, such as (i) inaccurate reports of medical history, (ii) missed doctor appointments, (iii) wrong medication used, (iv) increased rate of hospitalization, and also (v) decreased rates of adherence to chronic illness regiments. On the contrary, an increase in health literacy has proven to improve quality of life and reduce stress levels effectively.

However, Malaysia's health literacy, especially on non-communicable diseases, is still low, as Ting et al. (2019) reported. Moreover, Malaysian also has low awareness of non-communicable diseases such as CVD and obesity, where $43.5 \%$ of its population are overweight (Jaganathan et al., 2019). Another report by Ng (2018) stated that Malaysian score poorly in health literacy which led to ongoing obesity problems, the anti-vaccination movement, homebirth advocates, and predilection for health supplements. The lack of knowledge among Malaysians about health is the determinant of various health issues (Zhu, 2019). Her study also shows a high correlation between a low level of knowledge and susceptibility to health problems.

Although Malaysia is one of the countries that signed the Shanghai $2017 \mathrm{dec}-$ laration to commit to developing, implementing, and monitoring strategies for strengthening health literacy among Malaysians, the level of health literacy is still questionable Abdullah et al. (2020). According to Noman et al. (2019), few Malaysians work toward an online solution to increase health among citizens through mass education and prevention. Rahim et al. (2019) also supported this statement based on their online study on the engagement of health content in Malaysia. These studies stated that there should be a comprehensive update of health information among Malaysians.

A systematic review by Rajah et al. (2019) shows that among all the five studies that have been done in Malaysia, three of them displayed the prevalence of limited health literacy of more than $50 \%$ of the respondents assessed. As health literacy is essential to prevent further health problems, these issues can cause more significant health results that can cause more critical and fatal cases. A study by Yee et al. (2019) on the patient with chronic kidney disease found that majority (71\%) of the respondent have low health literacy and don't even want to admit to therapy mostly because they don't have information on the importance of the treatment. Another study by Abdullah et al. (2019) of the health literacy among diabetes patients shows that about $65.3 \%$ of the patients have a high prevalence of health literacy. They also suggested that a universal approach must be adopted to address the limited health 
literacy among Malaysians. Although there are alarming consents on Malaysian health literacy, the number of published papers on the field is limited in numbers (Abdullah et al., 2020).

Health is a crucial topic, but many studies, such as by Juvinyà-Canal et al. (2020) and Ibrahim et al. (2019), found that by providing relevant health information to young people, they can significantly improve their health and practice a healthy lifestyle. For the Malaysian education system, the health education that was published in the Malaysian textbook is only limited to reproductive and social health $(75 \%)$, diet (15\%), and first aid (10\%). Based on the Ng (2018) report, the syllabus used in Malaysia schools is not even the same as the other developed country. Many secondary school topics (age 13 to 17 ) are repetitive of the same three main content. Malaysian youth has found to be low on health literacy related to sexual and reproductive health (Qi \& Omar, 2020), mental health (Muhamad et al., 2017), body mass index, eating attitude, and physical activity (Dev \& Henry, 2016) and suitable fitness activity based on their health information (Mokmin \& Jamiat, 2020). As health literacy is critical to prevent health issues, Malaysian youth should equip themselves with the appropriate health information to make them ready to face the challenging adult world.

Bröder et al. (2017) also has pointed out that health literacy among youth is critical because, during this phase, the fundamental cognitive, physical and emotional development processes occur and develop health-related behaviors and skills. However, a recent study done by Juvinyà-Canal et al. (2020) proved that almost $93 \%$ of undergraduate students assessed have a low level of health literacy, and another study by Riiser et al. (2020) displayed the significant effect of the common health literacy among youth toward disease prevention. Hamzah et al. (2018) found that the level of health literacy among the Malaysian youth in their study was moderate $(\mathrm{M}=3.61, \mathrm{SD}=0.51$ on a scale of 5$)$. Another survey by Hassali et al. (2017) showed that the lack of health information caused the youth in their study to ignore their health condition and don't want to seek medical help. These reports should alert the decision-makers, health experts, and health-related system developers to find the best method to relay health information to these targeted users.

Chatbot has played significant roles in providing health information and is predicted to be a trend for the near future. According to a report by Holmes et al. (2018) and Nadarzynski et al. (2019), a health chatbot has the potential to increase access to healthcare information, improve the doctor-patient relationship, enable faster clinic-patient communication, manage the increasing demand for healthcare services, help to monitor medication adherence, and also help in keeping a person fit and healthy. Vaira et al. (2018) created a chatbot that functions to support women and families during pregnancy. The chatbot named Mamabot provides assistance and instructions in relevant situations, starting from the early pregnancy stage to pediatric treatment. Among other top health chatbots on the market is Youper by Youper (2020) that helps users take control of their emotional health. Babylon Health by Babylon Health UK (2020) provides health services with live video consultation from doctors. These technologies significantly alter healthcare delivery with a cost-effective solution and narrow the gap between health and 
well-being. However, according to several researchers, these assumptions still need further studies and validation.

A health chatbot can educate the users' behavior in deciding and taking action toward their healthy life. It will also encourage the users' motivation to make sure the quality of life is healthy and well managed. The health chatbot could also advise and help the medical practitioner provide the prevention tips of health issues. According to the Malaysian Healthcare Performance Unit, primary healthcare is served by public and private clinics. From 2011 to 2016, the health clinics density was around 32 clinics per 100,000 population (range 32.1-32.7 per 100,000 population). In parallel with this statistic, this shows how vital chatbot could get directly to the people via mobile application or online to help the user and medical practitioner educate and advise on health issues.

A chatbot is a part of AI technology that utilized the natural language processing algorithm to provide conversational experiences by mimicking how humans talk to each other. The first chatbot was developed by Joseph Weizenbaum in the 1960s and then got modernized in 2009 by a Chinese company that created the Wechat app (Kossler, 2017). Since then, the chatbot has become a necessity in several countries and has become the number one choice for marketers and employers to reduce tasks. However, Milne-Ives et al. (2020) study the effectiveness of 14 chatbots health-related chatbots in making conversation; for health, it showed mixed reviews. Chatbot agents indicate specific limitations to what they can offer, and further study should be done to evaluate the chatbot technology.

Nevertheless, the blooming of chatbot technology does not mean that the developers and designers of chatbot should neglect the rules of developing a good chatbot. The technical design, linguistic challenges, and emotional intelligence conversation should be designed carefully for a chatbot. According to Abd-Alrazaq et al. (2020), the chatbot should be evaluated based on usability, performance, responses, and esthetics. However, the experts expressed their caution of using the chatbot technology in health-related activities, as stated by John et al. (2020) in their study. It is crucial to evaluate the chatbot's design and functionality from the users' perspective because the developed chatbot's information can play a significant role in their health-related activities.

Laumer et al. (2020) used UTAUT2 to measure the user acceptance for disease diagnosis, and they also interviewed the users to know their acceptance of the chatbot. Their study showed that the intention to adopt the chatbot depends on how fast it will diagnose any symptom. Their interviews showed that adopting a chatbot assistant for disease diagnosis depends mainly on the chatbot's performance. The users also stated that the less effort needed for them to answer, the better the acceptance. The users also need facilitating conditions to assist in their diagnosis. Social influence, habit, data privacy, trust, compatibility, and performance expectancy are patients' expectations from using a chatbot assistant for disease diagnosis.

Thus, for a chatbot to provide relevant health information to increase health literacy, the acceptance, usefulness, and analytic study should be 
done to understand this technology's effect better. It is essential to address the low health literacy issues among youth in Malaysia, and chatbot technology can solve the problem. The UTAUT2 is also believed to be the most suitable theoretical lens to model health issues' adoption. Prakash and Das (2020) used the assessment to investigate the acceptance of patients seeking private mental healthcare services. Their study showed empirical evidence that the UTAUT2 constructs are vital in determining the users' decision to use a mental health chatbot. Their research also suggested that the hedonic aspect is the most critical in adding mental health therapy acceptance by a chatbot.

Considering all the facts above, this study attempts to explore the following objectives: (i) to evaluate the usefulness of the chatbot in making conversation with the real users, (ii) to assess the performance of the chatbot using the Google Analytics dashboard, and (iii) to evaluate the users' acceptance toward the chatbot technology to give health advice using the UTAUT2 instrument. The chatbot evaluation is essential to determine its usefulness, and the user's acceptance will be used to understand more from the user's perspective about chatbot. For the chatbot analysis assessment, the number of users session that has matched the intent and the exits percentages of the user query are used to measure the chatbot's performance. The result of this study will reveal the role of a health chatbot that could improve healthy life and ensure the proper physical activities are being done. The study is significant and relevant to the Fourth Industrial Revolution (4IR) and New Normal due to the Covid-19 pandemic that changed the norm activities worldwide. People will practice health education by using artificial intelligence health chatbot to ensure a healthy life is becoming more critical in their daily lives.

\section{Methodology}

The whole study evaluated a conversational health chatbot that the developer coined as Intelligent Health Advice Bot (IHAB). This technology is integrated into a fitness app that objectively gives users advice on health issues and fitnessrelated activities. The chatbot can be used individually and can also be accessible via Telegram and WhatsApp. The developed health chatbot application was reviewed by two professional medical doctors and an experienced instructional designer. The chatbot's intents and entities contain a knowledge repository of standard health information built based on health professionals' input.

To achieve the objectives of this study, several research questions should be answered. The first research question is "What is the evaluation of the chatbot in terms of its usefulness in providing recommendations to the targeted user." For this, we use a structured interview instrument. The second research question is "What is the analytics assessment of the chatbot, which answered using the Google Analytics analysis" and the last research question is "What is the user's acceptance toward the chatbot technology" and the UTAUT2 is as the instrument. This mixed-method 


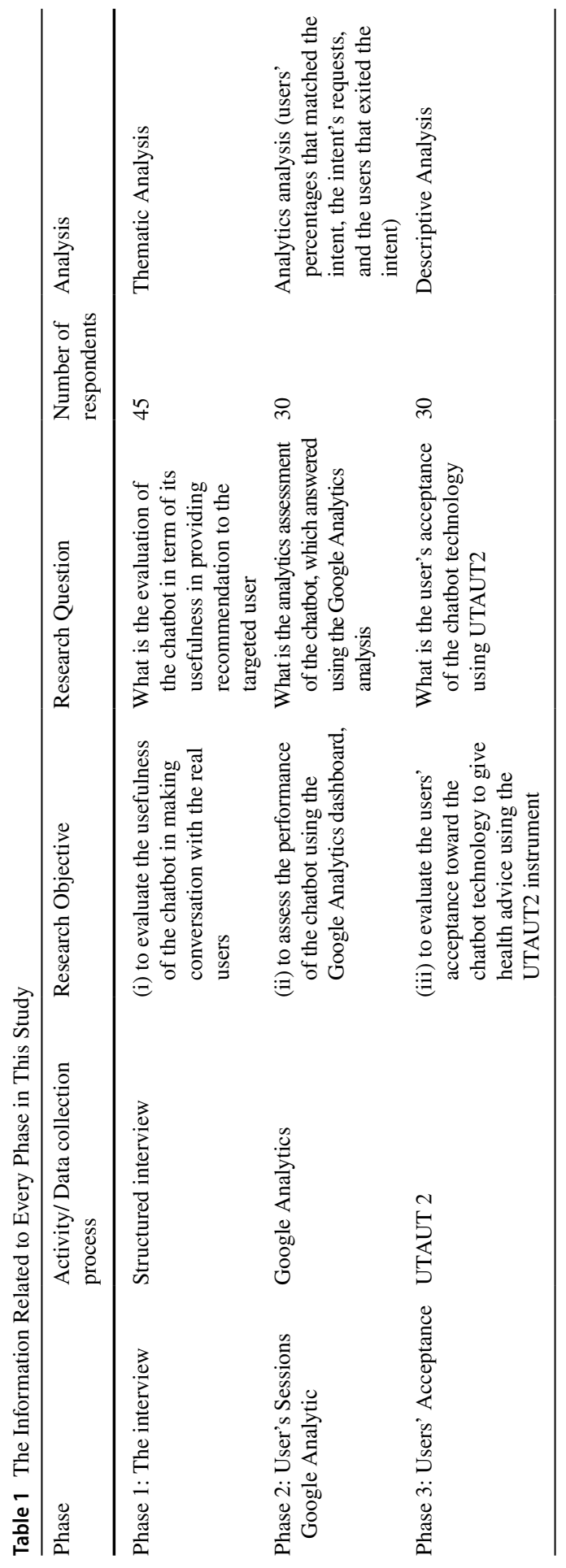


study combines both the quantitative and qualitative methods to address all the research questions.

The data collection procedure can be divided into there phases: (i) Phase 1: The interview, (ii) Phase 2: The Google Analytics, and (iii) Phase 3: The Users' Acceptance Study. Table 1 below described the phases, number of respondents, and instrument used in this study.

\subsection{Phase 1: The interview}

For the first objective, a structured interview was done with the actual users to test the chatbot's conversation in the real setting. The app's functionality was tested on 45 undergraduate students as the participants for this study in one local public university using the convenience sampling technique. The students were aged 20 to 23 years old and familiar with mobile app technology. The students attended the same course and have been exposed to chatbot technology before the experiment was done. The test ran for a month, and the respondents were asked to interact casually with the chatbot using the app link via a mobile platform. A structured interview was organized with the students. They were asked to test the app's functionality, ask a common health question, and communicate with the chatbot like an actual human would interact. The constructs of the interview were as the following: (i) the ability of the chatbot to answer health issues like a human, (ii) the power of the chatbot to give health and fitness advice, (iii) the added functionality that could improve the chatbot, and (iv) health information that they can obtain from the conversation with the chatbot.

\subsection{Phase 2: User's sessions google analytic}

The second objective was achieved by analyzing the chatbot's performance in responding to a user's queries. The purpose was to identify the chatbot's implementation in responding to the user's questions using the session analytics and the user's perception toward chatbot technology. The session analytics was provided in the Google Analytics dashboard to measure all users' percentages that matched the intent, the intent's requests, and the users that exited the intent. The invitation to test IHAB's sessions was distributed online. Then, the respondents participated in an online survey to measure their perception of the chatbot. The data collected from both instruments were analyzed in Google Analytics (user's sessions) and descriptive statistics (user's perception). Based on a study by Gay et al. (2011), a sample size of a minimum of 30 participants is accepted when the validity and reliability of the variables are high.

The chatbot sessions provided the data in Google Analytics that collected the big data of a user's conversations with the chatbot. Google Analytics data fulfilled the definition that the data are reliable to measure all users' percentages that matched the intent, the intent's requests, and the users that exited the intent. Google Analytics 
Table 2 The Seven-Point Likert Scale used in this Study

Performance Expectancy

Q1. I find health knowledge is helpful.

Q2. Using chatbot enables me to understand health better.

Q3. Using chatbots improves my knowledge of health.

Effort Expectancy

Q4. It is easy for me to improve my health knowledge when using a chatbot.

Q5. I find improving health knowledge is easy via chatbot.

Q6. Learning to improve my health using a chatbot is easy for me.

Attitude Toward Improving Health

Q7. Improving health via chatbot is fun.

Q8. I like to improve my health by using the chatbot.

Q9. Using a chatbot makes the health conversation more interesting.

Social Influence

Q10. People around me motivate me to improve health using the chatbot.

Q11. My friends introduced me to using health chatbot.

Q12. My friends have supported each other in using health chatbot.

Facilitating Conditions

Q13. I have the resources necessary to use the chatbot.

Q14. I know necessary to communicate with the chatbot.

Q15. A specific person (or group) is available for assistance with chatbot conversation difficulties.

Self Efficacy

Q16. I could use the chatbot if there is no one around to tell me what to do.

Q17. I could use the chatbot if I have a lot of time to complete the tasks.

Q18. I could use the chatbot if I could find helpful information when I got stuck.

Anxiety

Q19. I feel anxious about getting information via chatbot.

Q20. Using chatbot is intimidating to me.

Q21. I hesitate to use the chatbot for fear of making mistakes

Behavioural Intention to Use/Learn Chatbot

Q22. I plan to use a chatbot shortly.

Q23. I intend to learn more about using a chatbot soon.

Q24. I predict I will use/learn chatbot shortly.

is one of the available free tools that provide open quantitative data on chatbot usage that can be leveraged for continual chatbot improvements (Song et al., 2018).

\subsection{Phase 3: Users' acceptance}

The last objective was achieved by measuring how the factors that influence new technologies' perception are often undertaken using various incarnations of the UTAUT2 (Venkatesh et al., 2012). In this study, the "Behavioural Intention to Use/ Learn Chatbot" is added to the UTAUT2 construct suggested by Terblanche \& Cilliers (2020). A survey is conducted in this study to measure user perception toward chatbot technology. The survey instrument use the seven-point Likert scale; Strongly disagree (1); Disagree (2); Somewhat disagree (3); Neither agree nor disagree (4); Somewhat agree (5); Agree (6); Strongly agree (7) (Long et al., 2019). Table 2 shows the seven-point Likert's scale used in this study. 


\section{Performance Expectancy}

Q1. I find health knowledge is helpful.

Q2. Using chatbot enables me to understand health better.

Q3. Using chatbots improves my knowledge of health.

\section{Effort Expectancy}

Q4. It is easy for me to improve my health knowledge when using a chatbot.

Q5. I find improving health knowledge is easy via chatbot.

Q6. Learning to improve my health using a chatbot is easy for me.

\section{Attitude Toward Improving Health}

Q7. Improving health via chatbot is fun.

Q8. I like to improve my health by using the chatbot.

Q9. Using a chatbot makes the health conversation more interesting.

\section{Social Influence}

Q10. People around me motivate me to improve health using the chatbot.

Q11. My friends introduced me to using health chatbot.

Q12. My friends have supported each other in using health chatbot.

\section{Facilitating Conditions}

Q13. I have the resources necessary to use the chatbot.

Q14. I know necessary to communicate with the chatbot.

Q15. A specific person (or group) is available for assistance with chatbot conversation difficulties.

\section{Self Efficacy}

Q16. I could use the chatbot if there is no one around to tell me what to do.

Q17. I could use the chatbot if I have a lot of time to complete the tasks.

Q18. I could use the chatbot if I could find helpful information when I got stuck.

\section{Anxiety}

Q19. I feel anxious about getting information via chatbot.

Q20. Using chatbot is intimidating to me.

Q21. I hesitate to use the chatbot for fear of making mistakes

\section{Behavioural Intention to Use/Learn Chatbot}

Q22. I plan to use a chatbot shortly.

Q23. I intend to learn more about using a chatbot soon.

Q24. I predict I will use/learn chatbot shortly.

Fig. 1 IHAB Via Whatsapp And Telegram Apps

Data of user perception toward the IHAB application was collected from the Likert scale questionnaires. The process of conducting these tests using the chatbot application was carried out by invitation through several mediums, such as Whatsapp and e-mail applications, to over 30 respondents. The respondents for this study also came from a public university, and these students have been exposed to mobile technology before the experiment was carried out. Each invitation sent to run this test included proper instructions and steps to use the chatbot as an introductory guide for respondents to use the application. This test's respondents consisted of people who had online access to the IHAB application via mobile phone. Figure 1 shows the application of the chatbot via Whatsapp and Telegram. 


\section{Result}

\subsection{The evaluation of chatbot's usefulness for delivering health conversation}

The survey showed that most of the students (73.3\%) found that the chatbot could give health advice during the conversation they had with the app. The remaining respondents were not sure whether the app could answer all the questions. In the interview, they stated that the chatbot could not answer some of the problems. Being a conversational chatbot, it may not process certain types of questions. The result also showed a limitation to the extent of health questions the chatbot could answer; the respondents stated that it could only answer direct and straightforward questions. One respondent even stated that the chatbot could only answer 50\% of the questions asked. The majority of the respondents indicated that they were satisfied with the functionality, even though the chatbot did not reply to some queries well.

Regardless of the query and the consultation they had with the chatbot, the respondents did agree that the chatbot could provide new knowledge to them, mostly about health. They received further health information, such as calorie intake based on their fitness levels, the fitness exercise they should follow, and health literacy information. This group was then asked to propose additional functions that would make the chatbot more human-like in giving health advice. The students suggested a more comprehensive range of answers that allows the chatbot to answer different types of questions. They also suggested the chatbot be programmed to understand other conversational languages. An added link could also be integrated to improve user understanding of the health information provided by the chatbot.

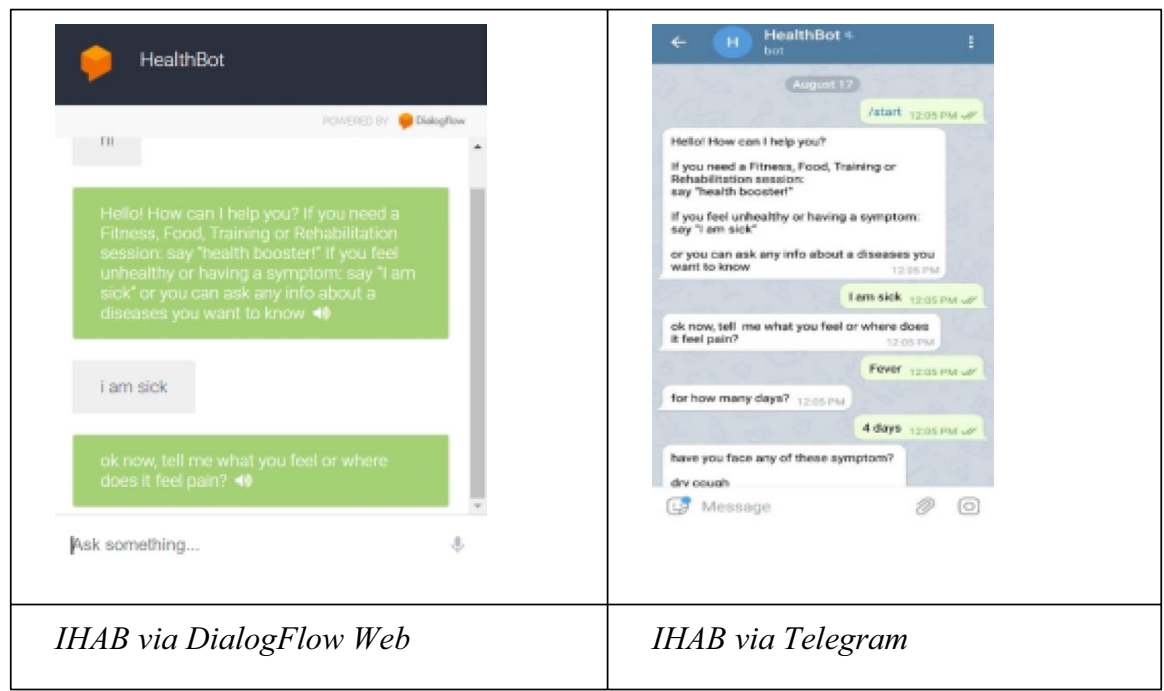

Fig. 2 The Google Analytics of the Sessions in the Last 30 Days 
66

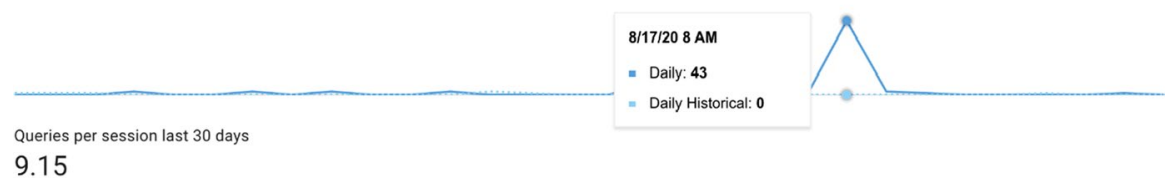

9.15

Fig. 3 The Most frequent Session Paths of IHAB

\subsection{The evaluation of IHAB's performance}

The users tested the IHAB application for approximately one month. Figure 2 shows the last 30 days of sessions, where 66 sessions that the users went through

Table 3 The Number of Sessions and Interactions for Each Intent in the Chatbot

\begin{tabular}{|c|c|c|c|}
\hline Intent & Sessions & Interactions & Exit \\
\hline Default Fallback Intent & 47 & 139 & $36.36 \%$ \\
\hline Default Welcome Intent & 63 & 115 & $19.70 \%$ \\
\hline Fitness & 18 & 21 & $7.58 \%$ \\
\hline Food & 21 & 32 & $4.55 \%$ \\
\hline No Match & 14 & 33 & $4.55 \%$ \\
\hline Advice & 31 & 36 & $1.52 \%$ \\
\hline KnowingSymptoms - fever - 7 day & 12 & 15 & $1.52 \%$ \\
\hline newcover & 8 & 10 & $1.52 \%$ \\
\hline thanks intent & 7 & 10 & $1.52 \%$ \\
\hline NovelCoronavirusDefinition & 4 & 4 & $1.52 \%$ \\
\hline Trainer 4 & 4 & 4 & $1.52 \%$ \\
\hline Assalamualaikum & 4 & 4 & $1.52 \%$ \\
\hline FoodAllergysymptoms & 2 & 3 & $1.52 \%$ \\
\hline HeartAttackDefinition & 2 & 2 & $1.52 \%$ \\
\hline exit & 1 & 1 & $1.52 \%$ \\
\hline KS - fever - 7 - covid - chronicdis - yes-w-b & 1 & 1 & $1.52 \%$ \\
\hline Foodtoavoid & 1 & 1 & $1.52 \%$ \\
\hline HypertensionComp - custom & 1 & 1 & $1.52 \%$ \\
\hline KS - fever - 7 - covid - chronicdis-fb - no & 1 & 1 & $1.52 \%$ \\
\hline Asthmarisk & 1 & 1 & $1.52 \%$ \\
\hline DiabetesDefinition & 1 & 1 & $1.52 \%$ \\
\hline Trainer & 1 & 1 & $1.52 \%$ \\
\hline
\end{tabular}


contributed to 9.15 queries for every session. From the graph below, the most active sessions were run at the end of the test period with 43 daily sessions.

Figure 3 shows the most frequent session paths that drive the user to use the IHAB application. Application usage was started with total percentages of sessions. IHAB's application was integrated with textbase applications such as Dialogflow Web and Telegram that can be used on multiple devices. The session path graph above shows that $85 \%$ of users followed "default welcome" intent at the second level of the path, and only $16 \%$ of users exited at the same point. There were $2 \%$ of no-match sessions that appeared at the third level of the path. However, there was no exit at this point, where the chatbot could not capture the user phrase, and the user was rephrasing the statement into the chatbot application. Table 3 shows a summary of the intent interactions.

The two intents with the most sessions and interactions in the IHAB application were "default welcome" intent and "default fallback" intent. These intents were the most used and matched because they were the most basic algorithm in this application. However, "knowing symptoms" intent recorded 37 sessions with 66 interactions, which showed that the user had used this application to get advice with the phrase "I am sick" which was directed from default welcome intent. Even though there was a default basic entry algorithm of intent, which was default fallback, fallback intent was the highest intent for user's exit, with default fallback at $36.36 \%$ and default welcome at $19.70 \%$ of exit. However, only $1.52 \%$ of exit was recorded in most of the intents of this IHAB application test.

\subsection{The users' technology acceptance of IHAB}

For the performance expectancy construct, more than $75 \%$ of IHAB's application's respondents agreed that the importance of health knowledge and the use of this application allow them to get a better understanding of health issues. The effort construct also showed that more than $70 \%$ of respondents agreed (somewhat agree, agree, and strongly agree) that it is easy for them to improve health knowledge by using and communicating with IHAB. They also found that the usage of this application was easy to enhance their learning toward health.

Both the attitude and self-efficiency constructs also had more than $70 \%$ approval in the survey, which displayed that the respondents had positive attitudes toward using a chatbot to increase their health knowledge. They also agreed that using the chatbot makes health conversation more interesting. Most of the respondents decided that they could use the chatbot without assistance or guidance. The use of new technology among respondents requires sufficient time, knowledge, and people around to enable them to use it well, as can be seen from the social construct, which showed that $58 \%$ of the respondents needed people to motivate them to use IHAB. This result was also related to the facilitation condition construct. Most of them also agreed that adequate resources to use the chatbot would make the technology better for long-term use. 


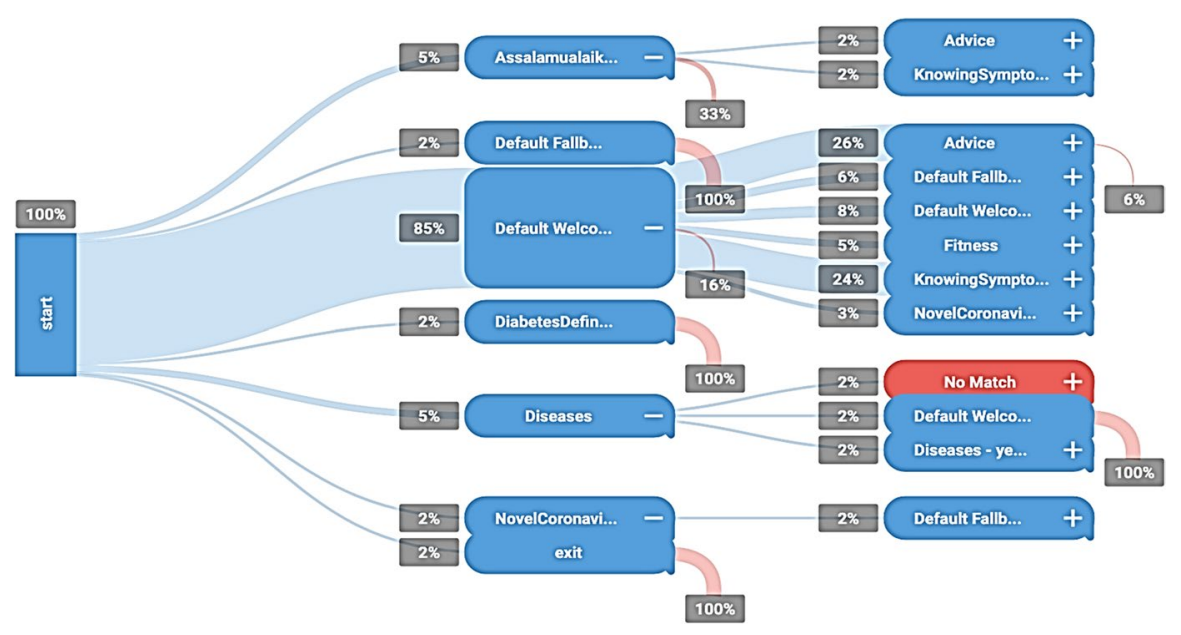

Fig. 4 Total percentage of agree scale (Strongly Agree, Agree, Somewhat agree)

The result also proved that only $50 \%$ of respondents agreed that they felt anxious about getting information via a chatbot. They were also concerned that using a chatbot was intimidating for them, and they hesitated to use the chatbot because of fear of making mistakes. The result showed that half of the respondents were worried about using IHAB. In contrast, another $30 \%$ of respondents disagreed that they felt anxious utilizing this application, and another $20 \%$ of respondents were undecided. The last construct, which was the behavioral intention, showed positive responses where $79 \%$ of respondents agreed to use a chatbot in the future and intended to learn more about using this application. Despite almost half of them feeling anxious about utilizing this application, there was a positive response to using IHAB technology as their health advisor.

There is currently a lack of knowledge regarding the factors that affect users' trust and acceptance toward chatbots technology (Følstad et al., 2018). The findings showed that the users have response positively toward the new technology by scoring high on "Performance Expectancy", "Effort Expectancy", "Attitude", "Self-efficiency", and "Behavioral Intention" with more than $70 \%$ of respondents agreed on these factors as shown in Fig. 4 below.

\section{Discussion}

This study introduced chatbot technology focusing on health advisory as a new technology in health literacy education. IHAB was developed using the Dialogflow platform by programming the intents that focused on various health issues. Additionally, this study conducted a structured interview, a survey questionnaire, and analytics analysis to evaluate the developed health chatbot. The findings showed that most of the students that tested the chatbot in a real setting with real health-related questions 
found that the chatbot successfully gave them the required answers. This was an excellent approach to increasing health literacy among undergraduate students as low literacy is a concern, as Omar Dev and Henry (2016) suggested. The undergraduate students represented today's youths who need health education learning materials to have adequate health literacy before encountering the working environment and prepare themselves for more significant health challenges.

The study also displayed the potential usage of chatbot technology as an excellent conversational agent to provide health advice to the new generation of users. The demand for health care services is at a maximum level this year since the outbreak of COVID-19. Many researchers and developers are now looking for ways to integrate the chatbot as conversational agents to support health-related activities, including increasing health literacy (Milne-Ives et al., 2020). IHAB's performance was evaluated by testing the application for 20 days and exploring and communicating regarding health issues.

The data were collected from the users' sessions, intents, and exits to measure the chatbot's performance in responding to the users' queries. The findings showed that users had more than one session and created more than 300 interactions in 66 sessions. Many transactions were recorded on Google Analytics. However, the factors for $37 \%$ of users to exit the sessions need to be reviewed. Each respondent explored IHAB actively to get advice from it with a phrase that matched the chatbot's intents on mobile devices. The analysis also showed that the chatbot had a low percentage of exits, where less than $37 \%$ of users exited the application.

Despite the users' anxiety with technology acceptance, there was a high behavioral intention to learn and use this application. From the results of this study, IHAB has the potential to be a health advisor by considering the facilitating conditions and diversifying the chatbot algorithm's intents to match users' queries. There is currently a lack of knowledge regarding the factors that affect users' trust and acceptance of chatbot technology (Følstad et al., 2018). The findings of user perception toward IHAB as a health advisor proved that users are positively responsive toward several technology acceptance factors, such as performance expectancy, effort expectancy, attitude, self-efficiency, and behavioral intention, with more than $70 \%$ of respondents agreeing on these factors.

Based on the findings, there was one session path that produced a $2 \%$ no-match in session. This occurred because of the system-default prompt that required users to repeat or retype their input when they returned a response to the Assistant. Dialogflow enforces a combined maximum of three no-match and no-input inputs. Once a conversation reaches three collection attempts, the Dialogflow agent will end the discussion with a default response. No-match information in Dialogflow is when one of the fallback intents is triggered. This is also aligned with the study by AbdAlrazaq et al. (2020), where they concluded that a chatbot should be evaluated based on usability, performance, responses, and esthetics.

For the acceptance of the technology by the targeted user, we can conclude that the users' perception of IHAB technology after using this application is that chatbot technology can improve their basic health knowledge, and provide early health advice if they are not well. This is aligned with the Laumer et al. (2020) findings that the chatbot's ability to provide health info can significantly affect the user's 
acceptance. The effectiveness of using this health chatbot technology has provided another paradigm shift in the health sector that allows users to get preliminary health advice before consulting professional medical practitioners or trainers.

\section{Implications}

This study has figured two categories of implications: IHAB as health advisor and the users' perception towards health chatbot technology. The intent of chatbot is the artificial intelligent chatbot that has been programmed with health data that will guide and advise the users until they find the right path on health issues in chat sessions. The phrase input and queries from the users will be analyzed by the systems and reply to the user's advice of health practice or related healthy advice. The effectiveness of using this health chatbot technology has provided another paradigm shift in the health sector that allows users to get initial advice on health before consulting a professional medical practitioner or trainers. The study also can be used as guidance for developer and educators to know the view, acceptance, and design of health advice chatbot that can provide health information when needed.

\section{Conclusion}

Health advice chatbot is a new technology that could integrate with many applications and platforms. Users can depend on the chatbot's intent to figure out medical information such as diseases, symptoms, and treatments on general illnesses as decision-support information before visiting a doctor. Should this study be developed for future studies, several things must be considered, such as diversifying the data's Dialogflow intents. Also, Health Chatbot was created and programmed for specific diseases or health objectives to give users a clear focus in using it. For health education, the chatbot has a tremendous potential to play a prominent role in educating users to increase their health literacy.

\section{References}

Abd-Alrazaq, A., Safi, Z., Alajlani, M., Warren, J., Househ, M., \& Denecke, K. (2020). Technical metrics used to evaluate health care Chatbots: Scoping review. Journal of Medical Internet Research, 22(6). https://doi.org/10.2196/18301.

Abdullah, A., Liew, S. M., Salim, H., Ng, C. J., \& Chinna, K. (2019). Prevalence of limited health literacy among patients with type 2 diabetes mellitus: A systematic review. PLoS One, 14(5), e0216402. https://doi.org/10.1371/journal.pone.0216402.t001.

Abdullah, A., May, L. S., Salim, H. S., Jenn, N. C., \& Chinna, K. (2020). Health literacy research in Malaysia: A scoping review. Sains Malaysiana, 49(5), 1021-1036. https://doi.org/10.17576/ jsm-2020-4905-07.

Babylon Health UK. (2020). Hello, how can I help you? https://www.babylonhealth.com/. Accessed 28 Jan 2020. 
Bröder, J., Okan, O., Bauer, U., Bruland, D., Schlupp, S., Bollweg, T. M., Saboga-Nunes, L., Bond, E., Sørensen, K., Bitzer, E. M., Jordan, S., Domanska, O., Firnges, C., Carvalho, G. S., Bittlingmayer, U. H., Levin-Zamir, D., Pelikan, J., Sahrai, D., Lenz, A., et al. (2017). Health literacy in childhood and youth: A systematic review of definitions and models. BMC Public Health, 17(1), 1-25. https:// doi.org/10.1186/s12889-017-4267-y.

Følstad, A., Nordheim, C. B., \& Bjørkli, C. A. (2018). What makes users trust a chatbot for customer service? an exploratory interview study. What makes users trust a chatbot for customer service? An exploratory interview study chatbots are software agents that interact with users through natural language. 11193(December), 194-208.

Gay, L. R., Mills, G. E., \& Airasian, P. (2011). Educational research: Competencies for analysis and applications (Tenth ed.).

Hamzah, S. R., Ismail, M., \& Mohd Nor, Z. (2018). Does attachment to parents and peers influence health literacy among adolescents in Malaysia? Kontakt, 20(4), e348-e355. https://doi.org/10.1016/j.konta kt.2018.10.006.

Hassali, M. A., Arief, M., Saleem, F., Khan, M. U., Ahmad, A., Mariam, W., Bheemavarapu, H., \& Syed, I. A. (2017). Assessment of attitudes and practices of young Malaysian adults about antibiotics use: A cross-sectional study. Pharmacy Practice, 15(2), 1-7. https://doi.org/10.18549/PharmPract.2017. 02.929 .

Holmes, S., Moorhead, A., Bond, R., Zheng, H., Coates, V., \& McTear, M. (2018). WeightMentor: A new automated chatbot for weight loss maintenance. 1-5. https://doi.org/10.14236/ewic/hci2018.103.

Ibrahim, N., Amit, N., Shahar, S., Wee, L. H., Ismail, R., Khairuddin, R., Siau, C. S., \& Safien, A. M. (2019). Do depression literacy, mental illness beliefs and stigma influence mental health helpseeking attitude? A cross-sectional study of secondary school and university students from B40 households in Malaysia. BMC Public Health, 19(Suppl 4), 1-8. https://doi.org/10.1186/ s12889-019-6862-6.

Jaganathan, R., Ramachandran, V., Ravindran, R., Suganthan, S., Ibrahim, N. A. B., Hisham, M. A. S. B. B., Akib, M. Z. B. M., \& Bin Zulkifli, M. R. (2019). Preclinical students' knowledge, attitude and practice towards obesity and overweight. Malaysian Journal of Public Health Medicine, 19(1), 41-46.

John, D. M., William, C. H., \& Ross, K. (2020). Clinical, legal, and ethical aspects of artificial intelligence-assisted conversational agents in Helath care. Law and Medicine, 324(6), 552-553. https:// doi.org/10.1001/jama.2020.2724.

Juvinyà-Canal, D., Suñer-Soler, R., Porquet, A. B., Vernay, M., Blanchard, H., \& Bertran-Noguer, C. (2020). Health literacy among health and social care university students. International Journal of Environmental Research and Public Health, 17(7), 1-10. https://doi.org/10.3390/ijerph17072273.

Kickbusch, I., Pelikan, J. M., Apfel, F., \& Tsouros, A. D. (2013). Health Literacy. In World Health Organization, 2013.

Kossler, V. (2017). The History of Chatbots What are chatbots ? Chatbot development over. https://onlim. com/en/the-history-of-chatbots/.

Laumer, S., Maier, C., \& Fabian, G. (2020). Chatbot acceptance in healthcare: Explaining user adoption of conversational agents for disease diagnosis. 27th European Conference on Information Systems Information Systems for a Sharing Society, ECIS 2019, 0-18.

Long, J., Yuan, J., \& Lee, H.-M. (2019). How to program a chatbot - an introductory project and student perceptions. Issues in Informing Science and Information Technology, 16, 001-031. https://doi.org/ $10.28945 / 4282$.

Milne-Ives, M., de Cock, C., Lim, E., Shehadeh, M. H., de Pennington, N., Mole, G., Normando, E., \& Meinert, E. (2020). The effectiveness of artificial intelligence conversational agents in health care: Systematic review. Journal of Medical Internet Research, 22(10). https://doi.org/10.2196/20346.

Mokmin, N. A. M., \& Jamiat, N. (2020). The effectiveness of a virtual fitness trainer app in motivating and engaging students for fitness activity by applying motor learning theory. Education and Information Technologies, September, 1-19. https://doi.org/10.1007/s10639-020-10337-7.

Muhamad, N. A., Roslan, N. M., Mahdi, A., Ithnain, N., Mustapha, N., Aliza, L. N., Melati, A. G. R., \& SM, S. (2017). Association between health risk behavior and suicidal ideation, continuous sadness and depression among Malaysian youth. Global Journal of Health Science, 10(1), 11. https://doi. org/10.5539/gjhs.v10n1p11.

Nadarzynski, T., Miles, O., Cowie, A., \& Ridge, D. (2019). Acceptability of artificial intelligence (AI)led chatbot services in healthcare: A mixed-methods study. Digital Health, 5, 1-12. https://doi.org/ $10.1177 / 2055207619871808$. 
Neter, E., \& Brainin, E. (2019). Association between health literacy, eHealth literacy, and health outcomes among patients with long-term conditions a systematic review. European Psychologist, 24(1), 68-81. https://doi.org/10.1027/1016-9040/a000350.

Ng, R. J. (2018). Poor score in health literacy. https://www.thestar.com.my/opinion/letters/2018/08/16/ poor-score-in-health-literacy.

Noman, M., Koo, A. C., Tee, S. H., \& Krishnan, M. (2019). Web elements for health promotion in Malaysia. Health Promotion International, 1-12. https://doi.org/10.1093/heapro/daz040.

Omar Dev, R. D., \& Henry, E. (2016). Effects of body mass index (Bmi), eating attitude and physical activity on bone health among undergraduate students in Malaysia. IJASOS- International E-Journal of Advances in Social Sciences, 2(5), 591. https://doi.org/10.18769/ijasos.91557.

Prakash, A. V., \& Das, S. (2020). Intelligent conversational agents in mental healthcare services : A thematic analysis of user perceptions. Pacific Asia Journal of the Association for Information Systems, 12(2), 1-34. https://doi.org/10.17705/1pais.12201.

Qi, C. K., \& Omar, N. (2020). Seeking sexual and reproductive health information among students at Universiti Utara Malaysia: A qualitative study on effective media use. Special Issue: The Sixth International Conference in Communication and Media - ICOME'18, 17-31. http://library1.nida.ac. th/termpaper6/sd/2554/19755.pdf.

Rahim, A. I. A., Ibrahim, M. I., Salim, F. N. A., \& Ariffin, M. A. I. (2019). Health information engagement factors in Malaysia: A content analysis of facebook use by the ministry of health in 2016 and 2017. International Journal of Environmental Research and Public Health, 16(4). https://doi.org/10. 3390/ijerph16040591.

Rajah, R., Hassali, M. A. A., \& Murugiah, M. K. (2019). A systematic review of the prevalence of limited health literacy in southeast Asian countries. Public Health, 167, 8-15. https://doi.org/10.1016/j. puhe.2018.09.028.

Riiser, K., Helseth, S., Haraldstad, K., Torbjørnsen, A., \& Richardsen, K. R. (2020). Adolescents' health literacy, health protective measures, and health-related quality of life during the Covid-19 pandemic. PLoS One, 15(8 august), 1-13. https://doi.org/10.1371/journal.pone.0238161.

Selden, C. R., Zorn, M., Ratzan, S. C., Parker, R. M., \& Compilers. (2000). National Library of Medicine Current Bibliographies in Medicine: Health Literacy. National Library of Medicine Current Bibliographies in Medicine: Health Literacy., January 2000, 33 p; 617 citations. https://doi.org/10.1017/ CBO9781107415324.004.

Song, M. J., Ward, J., Choi, F., Nikoo, M., Frank, A., Shams, F., Tabi, K., Vigo, D., \& Krausz, M. (2018). A process evaluation of a web-based mental health portal (WalkAlong) using google analytics. Journal of Medical Internet Research, 20(8), 1-10.

Terblanche, N., \& Cilliers, D. (2020). Factors that influence users' adoption of being coached by an Artificial Intelligence Coach. Philosophy of Coaching: An International Journal, 5(1), 61-70. https:// doi.org/10.22316/poc/05.1.06.

Ting, S.-H., Ho, J.-Y., \& Podin, Y. (2019). Receptivity towards breast cancer awareness materials: Message design and content. Journal of Humanities and Education Development, 1(1), 2581-8651. https://theshillonga.com/index.php/jhed.

Vaira, L., Bochicchio, M. A., Conte, M., Casaluci, F. M., \& Melpignano, A. (2018). Mama bot: A system based on ML and NLP for supporting women and families during pregnancy. ACM International Conference Proceeding Series, 273-277. https://doi.org/10.1145/3216122.3216173.

Venkatesh, V., Thong, J. Y. L., \& Xu, X. (2012). Consumer acceptance and use of information technology: Extending the unified theory of acceptance and use of technology. MIS Quarterly, 36(1), 157-178. https://doi.org/10.2307/41410412, https://www.jstor.org/stable/41410412.

Yee, C. W., Sxian, L. Z., Nurulfarahin, S., Yusop, M., Hida, N., \& Rodzi, M. (2019). Health literacy level among chronic kidney disease (CKD) patients at hospital Selama. Perak Medical Journal, 1(2), 40070.

Youper. (2020). Your emotional health assistant. https://www.youper.ai/. Accessed 28 Jan 2020.

Zhu, Y. (2019). Community based health promotion program for stroke in Malaysia. 2019 International Conference on Biomedical Sciences and Information Systems (ICBSIS 2019) Community, Icbsis, 91-95. https://doi.org/10.25236/icbsis.2019.020.

Publisher's note Springer Nature remains neutral with regard to jurisdictional claims in published maps and institutional affiliations. 